\title{
Hamburger and Stieltjes Moment Problems for Operators
}

\author{
L. Lemnete-Ninulescu \\ Department of Mathematics, "Politehnica” University of Bucharest, Splaiul Independenței, No. 313, 060042 Bucharest, Romania
}

Correspondence should be addressed to L. Lemnete-Ninulescu; luminita_lemnete@yahoo.com

Received 2 December 2013; Accepted 8 January 2014; Published 30 April 2014

Academic Editors: D. D. Hai and S. Pilipovic

Copyright (C) 2014 L. Lemnete-Ninulescu. This is an open access article distributed under the Creative Commons Attribution License, which permits unrestricted use, distribution, and reproduction in any medium, provided the original work is properly cited.

Solutions to some operator-valued, unidimensional, Hamburger and Stieltjes moment problems in this paper are given. Necessary and sufficient conditions on some sequences of bounded operators being Hamburger, respectively, Stieltjes operator-valued moment sequences are obtained. The determinateness of the operator-valued Hamburger and Stieltjes moment sequence is studied.

\section{Introduction}

A function $E(\lambda), a \leq \lambda \leq b$, is called a spectral function if

(a) $E(\lambda)$ is a bounded, positive operator,

(b) $E(\lambda) \leq E(\mu)$, for any $\lambda \leq \mu$,

(c) $E(\lambda+0)=E(\lambda)$,

(c') $E(a+0)=0$ and/or $E(b-0)=E(b)$ in case $a=-\infty$ and $/$ or $b=+\infty$.

The spectral function $E(\lambda)$ is called an orthogonal spectral function if every $E(\lambda)$ is an orthogonal projection [1, page 322].

(1) A sequence $\left\{A_{n}\right\}_{n=0}^{+\infty}$ of bounded self-adjoint operators, acting on an arbitrary, complex Hilbert space $\mathrm{H}$, subject on the condition $A_{0}=\mathrm{Id}_{\mathrm{H}}$, is called a Hamburger, unidimensional operator-valued moment sequence, if there exists an orthogonal spectral function $E(\lambda),-\infty \leq \lambda \leq+\infty$, such that $A_{n}=$ $\int_{-\infty}^{+\infty} \lambda^{n} \mathrm{~d} E(t), n=0,1,2, \ldots$, or

(2) a sequence $\left\{A_{n}\right\}_{n=0}^{+\infty}, A_{0}=\operatorname{Id}_{\mathrm{H}}$, of bounded selfadjoint operators is called a unidimensional operatorvalued Hamburger moment sequence, if there exists a positive operator-valued measure $F(\lambda),-\infty \leq \lambda \leq$ $+\infty$, measure generated by a spectral function, such that $A_{n}=\int_{-\infty}^{+\infty} \lambda^{n} \mathrm{~d} F(t), n=0,1,2, \ldots$.

A sequence $\left\{A_{n}\right\}_{n=0}^{+\infty}$ of bounded positive operators is called a Stieltjes unidimensional operator-valued moment sequence, if there exists a positive operator-valued measure $F(\lambda), 0 \leq \lambda \leq+\infty$, (generated by a spectral function) such that $A_{n}=\int_{0}^{+\infty} \lambda^{n} \mathrm{~d} F(\lambda), n=0,1,2, \ldots$. The passage from the integral representation (1) to an integral representation (2) is done, usually, by applying Naimark's dilation theorem, or modified forms of it as in [1].

In both cases (1) and (2), the operator-valued measures $E(\lambda)$ or $F(\lambda)$ are called the representing measures for the sequence $\left\{A_{n}\right\}_{n=0}^{+\infty}$. Necessary and sufficient conditions for representing scalar sequences or operator-valued sequences, in one or several variables, as Hamburger or Stieltjes moment sequences with respect to scalar, respectively, operatorvalued, positive measures, represent the subject of many outstanding papers such as [1-4]..., to quote only few of them.

In the present paper, in Section 3, we give a necessary and sufficient condition on a sequence of bounded, selfadjoint operators to be a Hamburger operator-valued, unidimensional moment sequence. In Section 4, we discuss the uniqueness of the representing measures of the operatorvalued Hamburger moment sequence both in (1) and (2) forms. In Section 5, we give some necessary and sufficient conditions on a sequence of positive operators to be a Stieltjes operator-valued, unidimensional moment sequence with respect to a positive, operator-valued measure. The positive representing measures in Sections 3 and 5 are obtained by applying Kolmogorov's theorem of decomposition of the positive definite kernels. 


\section{Preliminaries}

Let $t \in \mathrm{R}$ denote the real variable in the real Euclidean space; for $\mathrm{H}$ an arbitrary complex Hilbert space, $L(\mathrm{H})$ represents the algebra of bounded operators an $\mathrm{H}$; we denote with $\delta_{i}: \mathrm{N} \rightarrow$ $\{0,1\}$, the function

$$
\delta_{i j}= \begin{cases}1, & i=j, \\ 0, & i \neq j\end{cases}
$$

for $K$ a Hilbert space, $B(H, K)$ represents the set of bounded operators from $\mathrm{H}$ in $K$. We consider the $\mathrm{C}$-vector space of vectorial functions: $F=\{f:\{0,1, \ldots, n, \ldots,\} \rightarrow \mathrm{H}, f(\cdot)=$ $\sum_{n \in \mathrm{N}} \delta_{n} f(n), f$ with finite support, $\left.f(n) \in \mathrm{H}\right\}$. We define also the convolution $f * \delta_{1}$. $\in F$ as

$$
\left[f * \delta_{1}\right](n)=\sum_{k \in Z}\left(f(n-k) \cdot \delta_{1 k}\right), \quad n \in \mathrm{N}^{*},
$$

and make the convention: $f * \delta_{1}(0)=0_{\mathrm{H}}$. We have $f * \delta_{1}$. $\sum_{n \in \mathrm{N}} \delta_{(n+1)} f(n), f$ with finite support.

In Section 3, a necessary and sufficient condition on a sequence of self-adjoint operators to be a Hamburger operator-valued moment sequence is given. In Section 5, we give necessary and sufficient conditions on a sequence of positive operators to be a Stieltjes operator-valued moment sequence. In Section 4, the problem of the uniqueness of the represented measures in Sections 3 and 5 is studied. The representing measures in Sections 3 and 5 are obtained by applying Kolmogorov's theorem on decomposition of the positive kernels. Classical Kolmogorov's theorem for the decomposition of positive kernels is as follows:

"Let $\Gamma: S \times S \rightarrow L(H)$ be a nonnegativedefinite function where $S$ is an arbitrary set and $H$ a Hilbert space, namely, $\sum_{i, j=1}^{n}\left\langle\Gamma\left(s_{i}, s_{j}\right) x_{j}, x_{i}\right\rangle_{H} \geq$ 0 , for any finite number of points $s_{1}, \ldots, s_{n} \in S$ and any vectors $x_{1}, \ldots, x_{n} \in H$. In this case, there exists a Hilbert space $K$ (essentially unique) and a function $h: S \rightarrow B(H, K)$ such that $\Gamma(s, t)=$ $h(t)^{*} h(s)$ for any $s, t \in S$.”

We apply this theorem for a particular set $S$ and a particular positive-definite operator-valued function to give an integral representation as Hamburger operator-valued moment sequence and Stieltjes operator-valued moment sequence, respectively, to some sequences of self-adjoint and positive operators, respectively.

\section{An Operator-Valued Hamburger Moment Sequence Main Result}

Let $\Gamma=\left\{\Gamma_{n}\right\}_{n \in \mathrm{N}}$ be a sequence of bounded self-adjoint operators, acting on an arbitrary complex, separable Hilbert space; that is, $\Gamma_{n} \in L(\mathrm{H}), \Gamma_{n}=\Gamma_{n}^{*}$, for all $n \in \mathrm{N}, \Gamma_{0}=\mathrm{Id}_{\mathrm{H}}$, subject on the following conditions: for any finite vectors' sequence $\left\{x_{n}\right\}_{n \in I \subset N} \subset \mathrm{H}$, there exists another vector sequence $\left\{y_{n}^{\prime}\right\}_{n \in I \subset \mathrm{N}} \subset \mathrm{H}$ such that the following two equations are satisfied;

(A)

$$
\begin{aligned}
\sum_{p, q \in I}\left\langle\Gamma_{p+q+2}\left(x_{q}-y_{q}^{\prime}\right),\left(x_{p}-y_{p}^{\prime}\right)\right\rangle_{\mathrm{H}} & \\
& -2 \operatorname{Im} \sum_{p, q \in I}\left\langle\Gamma_{p+q+1}\left(x_{q}+y_{q}^{\prime}\right),\left(x_{p}-y_{p}^{\prime}\right)\right\rangle_{\mathrm{H}} \\
& +\sum_{p, q \in I}\left\langle\Gamma_{p+q}\left(x_{q}+y_{q}^{\prime}\right),\left(x_{p}+y_{p}^{\prime}\right)\right\rangle_{\mathrm{H}}=0 ;
\end{aligned}
$$

and for any finite vectors' sequence $\left\{x_{n}\right\}_{n \in I \subset \mathrm{N}} \subset \mathrm{H}$, there exists another vectors' sequence $\left\{y_{n}^{\prime \prime}\right\}_{n \in I \subset \mathrm{N}} \subset \mathrm{H}$ such that

(B)

$$
\begin{aligned}
\sum_{p, q \in I}\langle & \left.\Gamma_{p+q+2}\left(x_{q}-y_{q}^{\prime \prime}\right),\left(x_{p}-y_{p}^{\prime \prime}\right)\right\rangle_{\mathrm{H}} \\
& +2 \operatorname{Im} \sum_{p, q \in I}\left\langle\Gamma_{p+q+1}\left(x_{q}+y_{q}^{\prime \prime}\right),\left(x_{p}-y_{p}^{\prime \prime}\right)\right\rangle_{\mathrm{H}} \\
& +\sum_{p, q \in I}\left\langle\Gamma_{p+q}\left(x_{q}+y_{q}^{\prime \prime}\right),\left(x_{p}+y_{p}^{\prime \prime}\right)\right\rangle_{\mathrm{H}}=0 .
\end{aligned}
$$

Proposition 1. Let $\Gamma=\left\{\Gamma_{n}\right\}_{n \in N}$ be a sequence of bounded, selfadjoint operators, acting on an arbitrary complex, separable Hilbert space $H$, subject on the conditions: $\Gamma_{0}=I d_{H},(A)$ and (B) satisfied. The following statements are equivalent.

(i) We have:

$$
\sum_{n, m \in N}\left\langle\Gamma_{n+m} x_{m}, x_{n}\right\rangle_{H} \geq 0
$$

for all sequences $\left\{x_{n}\right\}_{n} \in H$ with finite support.

(ii) There exists a positive operator-valued measure $E_{A}$ (spectral function) defined on $\operatorname{Bor}(R)$ such that

$$
\Gamma_{n}=\int_{-\infty}^{+\infty} t^{n} \mathrm{~d} E_{A}(t), \quad n=0,1,2, \ldots, k, \ldots
$$

Proof. When $F=\{f:\{0,1, \ldots, n, \ldots\} \rightarrow \mathrm{H}, f(\cdot)=$ $\sum_{n \in\{0,1, \ldots, n, \ldots\}} \delta_{n} f(n), f$ with finite support $\}$ is the C-vector space of functions defined on $\mathrm{N}$ with vectorial values, we consider the kernel $\Gamma$ as a double indexed, symmetric one:

$$
\begin{gathered}
\Gamma:\{0,1, \ldots, n, \ldots,\} \times\{0,1, \ldots, n, \ldots,\} \rightarrow B(\mathrm{H}) \\
\Gamma_{n+m}=\Gamma(n, m) .
\end{gathered}
$$

With the aid of $\Gamma$, we introduce the Hermitian, square positive functional $\Lambda^{\Gamma}: F \times F \rightarrow C, \Lambda^{\Gamma}(f, g)=$ $\sum_{m, n \in\{0,1, \ldots, n, \ldots,\}}\left\langle\Gamma_{n+m} f(m), g(n)\right\rangle_{\mathrm{H}}$. From property (i) of the kernel $\Gamma$, as well as from the properties of the scalar product in $H, \Lambda^{\Gamma}$ satisfies the following conditions.

$\left(1^{0}\right) \Lambda^{\Gamma}$ is C-linear in the first argument.

$\left(2^{0}\right) \overline{\Lambda^{\Gamma}(f, g)}=\Lambda^{\Gamma}(g, f)$, for all $f, g \in F$. 
$\left(3^{0}\right) \Lambda^{\Gamma}(f, f) \geq 0$, for all $f \in F$; and, moreover, being a Hermitian square, positive functional on $F \times F, \Lambda^{\Gamma}$ satisfies the Cauchy-Buniakovski-Schwarz inequality, respectively:

$$
\left|\Lambda^{\Gamma}(f, g)\right| \leq \Lambda^{\Gamma}(f, f)^{1 / 2} \Lambda^{\Gamma}(g, g)^{1 / 2}, \quad \forall f, g \in F .
$$

Also, from the construction of the Hermitian functional $\Lambda^{\Gamma}$ and the symmetry of the kernel $\Gamma,\left(\Gamma_{n+1+m}=\Gamma(n+1, m)=\right.$ $\left.\Gamma(n, m+1)=\Gamma_{n+m+1}\right)$, the functional $\Lambda_{\mathrm{N}}^{\Gamma}$ satisfies the equalities:

$\left(5^{0}\right)$

$$
\Lambda^{\Gamma}\left(f * \delta_{1,}, g\right)=\Lambda^{\Gamma}\left(f, g * \delta_{1}\right), \quad \forall f, g \in F .
$$

With these assumptions, $F \ni f \rightarrow\langle f, f\rangle_{F}^{1 / 2}=\Lambda^{\Gamma}(f, f)^{1 / 2}$ is a seminorm on $F$. Let $S$ be the subset in $F$, defined as $S=\left\{f \in F\right.$ with $\left.\Lambda^{\Gamma}(f, f)=0\right\}$. If follows, using the CauchyBuniakovski-Schwarz inequality, that if $f_{1}, f_{2} \in S$, we have also $\Lambda^{\mathrm{\Gamma}}\left(\alpha_{1} f_{1}+\alpha_{2} f_{2}, \alpha_{1} f_{1}+\alpha_{2} f_{2}\right)=0$; that is, $S \subset F$ is a vector subspace in $F$. We consider the separated completion space of $F$ with respect to $S$; that is, in this case, the quotient completion space $K=\overline{F / S}^{\|\cdot\|_{\Gamma}}$. Obviously, $K$ is a Hilbert space with the usual norm in the completion Hilbert space and $D=F / S$ is a dense subspace of it (i.e., $\|\widehat{h}\|_{K}=\lim _{n}\left\|h_{n} x\right\|=$ $\lim _{n}\left\langle\delta_{n} x+S, \delta_{n} \cdot x+S,\right\rangle_{F}^{1 / 2}$, where $h x=\left\{h_{n} x\right\}$ is a Cauchy sequence of elements in $F / S$ and $\left.h_{n} x=\widehat{\delta_{n} \cdot x}=\delta_{n} \cdot x+S\right)$. The Hilbert space $K$ is uniquely defined and it is also described as $K=V \operatorname{Ran} h_{n} x$, (the closed linear span of the ranges of the operators $\left.h_{n}: \mathrm{H} \rightarrow K, h_{n} x=\widehat{\delta_{n} \cdot x}=\delta_{n} x+S\right)$. From Kolmogorov's decomposition theorem of positively defined kernels, with the above construction, the decompositions $\Gamma_{n+m}=\Gamma(n, m)=h_{n}^{*} h_{m}$ hold for any $n, m \in \mathrm{N}$. Let us consider the densely defined subspace of $K, D=F / S=\sum_{n \in I \subset \mathrm{N}} h_{n} x_{n}$, $I$ finite $x_{n} \in \mathrm{H}, D \subset K$, and the operator $A: D \rightarrow D$, defined by $A\left(\sum_{n \in I \subset N} h_{n} x_{n}\right)=\sum_{n \in I \subset N} h_{n+1} x_{n}$. We prove that $A$ is correctly defined. Consequently, we consider the elements $\sum_{m \in J \subset \mathrm{N}} h_{m} x_{m}=\sum_{m \in J \subset \mathrm{N}}\left(\delta_{m} \cdot x_{m}+S\right)$ and $\sum_{n \in I \subset \mathrm{N}} h_{n} x_{n}=$ $\sum_{n \in I \subset \mathrm{N}}\left(\delta_{n} \cdot x_{n}+S\right)$ such that $\sum_{n \in I \subset \mathrm{N}} \delta_{n} \cdot x_{n}-\sum_{m \in J \subset \mathrm{N}} \delta_{m} \cdot x_{m} \in$ $S$ and show that $A\left(\sum_{n \in I \subset N} h_{n} x_{n}\right)=A\left(\sum_{m \in I \subset N} h_{m} x_{m}\right)$. The above equality is the same as the equality: $\sum_{n \in I \subset N} h_{n+1} x_{n}=$ $\sum_{m \in J \subset N} h_{m+1} x_{m}$ (modulo $S$ ). Indeed, from $\left(5^{0}\right)$, we have

$$
\begin{aligned}
& \left|\Lambda^{\Gamma}\left(\sum_{n} \delta_{n+1} \cdot x_{n}-\sum_{m} \delta_{m+1} \cdot x_{m}, \sum_{n} \delta_{n+1} \cdot x_{n}-\sum_{m} \delta_{m+1} \cdot x_{m}\right)\right| \\
& =\left|\Lambda^{\Gamma}\left(\sum_{n} \delta_{n} \cdot x_{n}-\sum_{m} \delta_{m} \cdot x_{m}, \sum_{n} \delta_{n+2} \cdot x_{n}-\sum_{m} \delta_{m+2} \cdot x_{m}\right)\right| \\
& \leq \Lambda^{\Gamma}\left(\sum_{n} \delta_{n} \cdot x_{n}-\sum_{m} \delta_{m} \cdot x_{m}, \sum_{n} \delta_{n} \cdot x_{n}-\sum_{m} \delta_{m} \cdot x_{m}\right)^{1 / 2} \\
& \cdot \Lambda^{\Gamma}(l, l)^{1 / 2}=0,
\end{aligned}
$$

where $l=\sum_{n} \delta_{n+2} \cdot x_{n}-\sum_{m} \delta_{m+2} . x_{m}$. From the above definition, we have $\left(6^{0}\right)$

$$
\begin{aligned}
\langle A x, y\rangle_{K} & =\left\langle A\left(\sum_{n \in I \subset \mathrm{N}} h_{n} x_{n}\right), \sum_{m \in J \subset \mathrm{N}} h_{m} y_{m}\right\rangle_{K} \\
& =\left\langle\sum_{n \in I \subset \mathrm{N}} h_{n+1} x_{n}, \sum_{m \in J \subset \mathrm{N}} h_{m} y_{m}\right\rangle_{K} \\
& =\left\langle\sum_{m, n \in I \cup J \subset \mathrm{N}} h_{m}^{*} h_{n+1} x_{n}, y_{m}\right\rangle_{K} \\
& =\sum_{m, n}\left\langle\Gamma_{m+n+1} x_{n}, y_{m}\right\rangle_{\mathrm{H}} \\
& =\sum_{m, n}\left\langle\Gamma_{n+m+1} x_{n}, y_{m}\right\rangle_{\mathrm{H}} ;
\end{aligned}
$$

and also

$\left(7^{0}\right)$

$$
\begin{aligned}
\langle x, A y\rangle_{K} & =\left\langle\sum_{n \in I \subset \mathrm{N}} h_{n} x_{n}, A\left(\sum_{m \in J \subset \mathrm{N}} h_{m} y_{m}\right)\right\rangle_{K} \\
& =\left\langle\sum_{n \in I \subset \mathrm{N}} h_{n} x_{n}, \sum_{m \in J \subset \mathrm{N}} h_{m+1} y_{m}\right\rangle_{K} \\
& =\left\langle\sum_{m, n \in I \cup J \subset \mathrm{N}} h_{m+1}^{*} h_{n} x_{n}, y_{m}\right\rangle_{K} \\
& =\sum_{m, n}\left\langle\Gamma_{m+1+n} x_{n}, y_{m}\right\rangle_{\mathrm{H}} \\
& =\sum_{m, n}\left\langle\Gamma_{n+m+1} x_{n}, y_{m}\right\rangle_{\mathrm{H}} .
\end{aligned}
$$

From $\left(6^{0}\right)$ and $\left(7^{0}\right)\langle A x, y\rangle_{K}=\langle x, A y\rangle_{K}$ for $x, y \in D$ arbitrary; we infer that $A$ is a densely defined, symmetric operator. We prove that $A$ has equal deficiency indices in $K$; consequently $A$ 's Cayley transform is a partial isometry on $K_{+}=R\left(A+\mathrm{i} I_{K}\right)$ with values in $K_{-}=R\left(A-\mathrm{i} I_{K}\right)$. Indeed, let $K_{ \pm}=R\left(A \pm \mathrm{i} I_{K}\right)=V_{n \in I_{\text {fnitice }}}\left(h_{n+1} x_{n} \pm \mathrm{i} h_{n} x_{n}\right), x_{n} \in \mathrm{H}$ arbitrary, be the ranges in $K$ of the operators $\left(A \pm \mathrm{iI}_{K}\right)$. We prove that $K_{ \pm}$are vector subspaces in $K$. For this request, we consider the elements $f_{ \pm}=\sum_{n \epsilon_{1} \text { finitec }}\left(h_{n+1} x_{n} \pm \mathrm{i} h_{n} x_{n}\right)$, $g=\sum_{n \in I_{2} \text { finitec }}\left(h_{n+1} y_{n} \pm \mathrm{i} h_{n} y_{n}\right)$ in $K_{ \pm}$and $\alpha, \beta \in \mathrm{C}$ arbitrary. Let us define the elements:

$$
\begin{aligned}
\left\{\widetilde{x}_{n}\right\}_{n \in I_{1} \cup I_{2}=I}, & \widetilde{x}_{n}= \begin{cases}x_{n}, & n \in I_{1}, \\
0_{\mathrm{H}}, & n \in I_{2}-\left(I_{1} \cap I_{2}\right),\end{cases} \\
\left\{\widetilde{y}_{n}\right\}_{n \in I_{1} \cup I_{2}} & \\
\widetilde{y}_{n} & = \begin{cases}y_{n}, & n \in I_{2}, \\
0_{\mathrm{H}}, & n \in I_{1}-\left(I_{1} \cap I_{2}\right),\end{cases}
\end{aligned}
$$

also the elements $\tilde{f}_{ \pm}=\sum_{n \epsilon_{1} \text { finitec N }}\left(h_{n+1} \widetilde{x}_{n} \pm \mathrm{i} h_{n} \widetilde{x}_{n}\right)$, $\tilde{g}=\sum_{n \in I_{2} \text { finitec }}\left(h_{n+1} \widetilde{y}_{n} \pm \mathrm{i} h_{n} \tilde{y}_{n}\right)$ in $K_{ \pm}$and $\alpha, \beta \in \mathrm{C}$. It results 
that $f-\tilde{f}=0_{K} ; g-\widetilde{g}=0_{K}$ and $\left[\alpha \widetilde{f}_{ \pm}+\beta \widetilde{g}_{ \pm}\right]=\left[\alpha f_{ \pm}+\beta g_{ \pm}\right]=$ $\sum_{n \in I_{1} \cap I_{2}}\left[h_{n+1}\left(\alpha \widetilde{x}_{n}\right)+\mathrm{i} h_{n}\left(\alpha \tilde{x}_{n}\right)+h_{n+1}\left(\beta \tilde{y}_{n}\right) \pm \mathrm{i} h_{n}\left(\beta \tilde{y}_{n}\right)\right]+$ $\sum_{n \in I_{1}-\left(I_{1} \cap I_{2}\right)}\left[h_{n+1}\left(\alpha \tilde{x}_{n}\right)+\mathrm{i} h_{n}\left(\beta \tilde{y}_{n}\right)+h_{n+1}\left(\beta \tilde{y}_{n}\right) \pm \mathrm{i} h_{n}\left(\alpha \tilde{x}_{n}+\right.\right.$ $\left.\beta \tilde{y}_{n}\right]+\sum_{n \in I_{2}-\left(I_{1} \cap I_{2}\right)}\left[h_{n+1}\left(\alpha \tilde{x}_{n}\right)+\beta \tilde{y}_{n} \pm \mathrm{i} h_{n}\left(\alpha \tilde{x}_{n}\right)+\beta \tilde{y}_{n}\right)=$ $\sum_{n \in I_{1} \cap I_{2}}\left[h_{n+1}\left(\alpha \tilde{x}_{n}+\beta \tilde{y}_{n}\right) \pm \mathrm{i} h_{n}\left(\alpha \tilde{x}_{n}\right)+\beta \tilde{y}_{n}\right] \in K_{ \pm}$. Because $A$ is a symmetric operator, it results also that $R\left(A \pm \mathrm{i} I_{K}\right)=K_{ \pm}$are closed subspaces in $K$. We prove that, in conditions (A) and (B) for the kernel $\Gamma$, we have $K_{+}=R\left(A+\mathrm{i} I_{K}\right)=R\left(A-\mathrm{i} I_{K}\right)=K_{-}$. Indeed, given an arbitrary element $f=\sum_{n \in I_{\text {finite }} \subset \mathrm{N}}\left(h_{n+1} x_{n}+\mathrm{i} h_{n} x_{n}\right)$ we look for an element $g \in K_{-}=R\left(A-\mathrm{i} I_{K}\right), g=\sum_{n \in I_{\text {finite }} \mathrm{CN}}\left(h_{m+1} y_{m}^{\prime}-\mathrm{i} h_{m} y_{m}^{\prime}\right)$ such that $(f-g)=0_{K}$. For a construction of the elements $\left\{\widetilde{x_{n}}\right\}_{n \in I_{1} \cup I_{2}=I},\left\{\widetilde{y_{n}}\right\}_{n \in I_{1} \cup I_{2}=I} \subset \mathrm{H}$ like the previous one, we have $(f-g)=\sum\left[h_{n+1}\left(\tilde{x}_{n}-\tilde{y}_{n}^{\prime}\right)+\mathrm{i} h_{n}\left(\tilde{x}_{n}+\tilde{y}_{n}^{\prime}\right)\right]=$ $0_{K} \Leftrightarrow \sum_{p, q \in I} \Gamma_{p+q}\left[\sum_{n} \delta_{(n+1) .}\left(\widetilde{x}_{n}-\tilde{y}_{n}^{\prime}\right)+\mathrm{i} \delta_{n .}\left(\tilde{x}_{n}-\tilde{y}_{n}^{\prime}\right)\right](q)$, $\left[\sum_{n} \delta_{(n+1) .}\left(\tilde{x}_{n}-\tilde{y}_{n}^{\prime}\right)+\mathrm{i} \delta_{n .}\left(\tilde{x}_{n}-\tilde{y}_{n}^{\prime}\right)\right](p)>_{\mathrm{H}}=0 \Leftrightarrow$ $\sum_{p, q \in I, p, q \geq 1} \Gamma_{p+q}\left[\left(\tilde{x}_{q-1}-\tilde{y}_{q-1}^{\prime}\right)+\mathrm{i}\left(\tilde{x}_{q}+\tilde{y}_{q}^{\prime}\right)\right],\left(\tilde{x}_{p-1}-\tilde{y}_{p-1}^{\prime}+\right.$ $\mathrm{i}\left(\tilde{x}_{p}+\tilde{y}_{p}^{\prime}\right)>_{\mathrm{H}}=0_{K} \Leftrightarrow \sum_{p, q \geq 0}\left\langle\Gamma_{p+q+2}\left(\tilde{x}_{q}-\tilde{y}_{q}^{\prime}\right),\left(\tilde{x}_{p}-\tilde{y}_{p}^{\prime}\right)\right\rangle_{\mathrm{H}}-$ $2 \operatorname{Im} \sum_{p, q \in I}\left\langle\Gamma_{p+q+1}\left(\tilde{x}_{q}+\tilde{y}_{q}^{\prime}\right),\left(\tilde{x}_{p}-\tilde{y}_{p}^{\prime}\right)\right\rangle_{\mathrm{H}}+\sum_{p, q \in I}\left(x_{q}+\right.$ $\left.\tilde{y}_{q}^{\prime}\right),\left(\tilde{x}_{p}+\tilde{y}_{p}^{\prime}\right)>_{\mathrm{H}}=0_{K}$. According to condition (A), on the kernel $\left\{\Gamma_{n}\right\}_{n}$, such an element exists. We have $K_{+} \subset K_{-}$. Conversely, let $f \in K_{-}, f=\sum_{n \in I}\left(h_{n+1} x_{n}-\mathrm{i} h_{n} x_{n}\right)$; we search for an element $g \in K_{+}, g=\sum_{n \in I}\left(h_{n+1} \widetilde{y}_{n}^{\prime \prime}+\mathrm{i} h_{n} \widetilde{y}_{n}^{\prime \prime}\right)$ with the property that $(f-g)=0_{K}$. Consequently, we have to find an element $g \in K_{+}$such that $\sum\left[\delta_{(n+1) .}\left(\widetilde{x}_{n}-\widetilde{y}_{n}^{\prime}\right)+\mathrm{i} h_{n}\left(\widetilde{x}_{n}+\widetilde{y}_{n}^{\prime}\right)\right]=$ $0_{K} \Leftrightarrow \sum_{p, q \in I} \Gamma_{p+q}\left[\sum_{n} \delta_{(n+1) .}\left(\tilde{x}_{n}-\tilde{y}_{n}^{\prime \prime}\right)-\mathrm{i} \delta_{n .}\left(\tilde{x}_{n}+\tilde{y}_{n}^{\prime \prime}\right)\right](q)$, $\left.\sum_{n} \delta_{(n+1) .}\left(\tilde{x}_{n}-\tilde{y}_{n}^{\prime \prime}\right)-\mathrm{i} \delta_{n .}\left(\tilde{x}_{n}+\tilde{y}_{n}^{\prime \prime}\right)\right](p)>_{\mathrm{H}}=0_{K} \Leftrightarrow$ $\sum_{p, q \in I}\left\langle\Gamma_{p+q+2}\left(\tilde{x}_{q}-\tilde{y}_{q}^{\prime \prime}\right),\left(\tilde{x}_{p}-\tilde{y}_{p}^{\prime \prime}\right)\right\rangle_{\mathrm{H}}+2 \operatorname{Im} \sum_{p, q \in I}\left\langle\Gamma_{p+q+1}\right.$ $\left.\left(\tilde{x}_{q}+\tilde{y}_{q}^{\prime \prime}\right),\left(\tilde{x}_{p}-\tilde{y}_{p}^{\prime \prime}\right)\right\rangle_{\mathrm{H}}+\sum_{p, q \in I}\left\langle\Gamma_{p+q}\left(\tilde{x}_{q}+\tilde{y}_{q}^{\prime \prime}\right),\left(\tilde{x}_{p}+\tilde{y}_{p}^{\prime \prime}\right)\right\rangle_{\mathrm{H}}=$ 0 . We prove with these computations that $K_{+}=R\left(A+\mathrm{i} I_{K}\right)=$ $K_{-}=R\left(A-\mathrm{i} I_{K}\right) \subset K$. That is $\operatorname{dim} K_{+}^{\perp}=\operatorname{dim} K_{-}^{\perp} \Rightarrow A^{\prime}$ 's Cayley transform has equal deficiency indices and $\Rightarrow \quad A$ admits a self-adjoint extension $\widetilde{A}$. Let $E_{A}$ be the spectral measure of the self-adjoint operator $\widetilde{A}$. Because $h_{m} x=A^{m}\left(h_{0} x\right)$ for all $x \in \mathrm{H}$ and $\Gamma_{m} x=h_{0}^{*} h_{m} x$, it results that $\Gamma_{m} x=h_{0}^{*} A^{m} h_{0} x$, for all $x \in \mathrm{H}$ and the integral representations $\Gamma_{m} x=h_{0}^{*} \int_{\mathrm{R}} t^{m} \mathrm{~d} E_{A}(t) h_{0} x$, for all $x \in \mathrm{H}$, for all $m \in \mathrm{N}$. We consider the positive operator-valued measure $F_{A}(t)=h_{0}^{*} E_{A} h_{0}$. With respect to this positive operatorvalued measure, we have $\Gamma_{m} x=h_{0}^{*} A^{m} h_{0} x=\int_{\mathrm{R}} t^{m} \mathrm{~d} F_{A}(t) x$, for all $x \in \mathrm{H}$ and all $m \in \mathrm{N}$. That is $\Gamma_{m}=\int_{\mathrm{R}} t^{m} \mathrm{~d} F_{A}(t)$, for all $m \in \mathrm{N}$, the required Hamburger moment integral representations.

Conversely. If the terms $\left\{\Gamma_{m}\right\}_{m}$ admit the integral representations $\Gamma_{m}=\int_{-\infty}^{+\infty} t^{m} \mathrm{~d} F_{A}(t)$, for all $m=0,1,2, \ldots$, for a positive operator-valued measure on $\mathrm{R}$, we have

$$
\begin{aligned}
\sum_{n, m}\left\langle\Gamma_{n+m} x_{m}, x_{n}\right\rangle_{\mathrm{H}} & \\
& =\sum_{n, m}\left\langle\int_{-\infty}^{+\infty} t^{n+m} \mathrm{~d} F_{A}(t) x_{m}, x_{n}\right\rangle_{\mathrm{H}}
\end{aligned}
$$

$$
\begin{aligned}
& =\int_{-\infty}^{+\infty} \mathrm{d}\left(\left\langle\sum_{m} t^{m} F_{A}^{1 / 2}(t) x_{m}, \sum_{n} t^{n} F_{A}^{1 / 2}(t) x_{n}\right\rangle_{\mathrm{H}}\right) \\
& =\int_{\mathrm{R}} \mathrm{d}\left(\left\|F_{A}^{1 / 2} \sum_{n} t^{n} x_{n}\right\|^{2}\right) \geq 0,
\end{aligned}
$$

as it is required by (i).

\section{About the Uniqueness of the Hamburger Operator-Valued Moment Sequences' Representations}

Let us consider a sequence of bounded operators $\left\{A_{n}\right\}_{n \in \mathrm{N}}$, $A_{n} \in L(\mathrm{H})$, subject on the condition $A_{n}=A_{n}^{*}, A_{0}=\mathrm{Id}_{\mathrm{H}}$, $n=0,1,2, \ldots, \mathrm{H}$ an arbitrary complex Hilbert space. For the sequence $\left\{A_{n}\right\}_{n \in \mathrm{N}}$, we get two operator-valued integral representing measures (or spectral functions), $E_{A}, E_{B}$ : $\operatorname{Bor}(\mathrm{H}) \rightarrow A(\mathrm{H})$, that is,

$$
A_{n}=\int_{-\infty}^{+\infty} t^{n} \mathrm{~d} E_{A}(t)=\int_{-\infty}^{+\infty} t^{n} \mathrm{~d} E_{B}(t)
$$

for all $n=0,1,2, \ldots$. The operator-valued measures allow us to define the scalar measures $\mu^{x}, \nu^{x}: \operatorname{Bor}(\mathrm{R}) \rightarrow[0,+\infty]$, $\mu^{x}(D)=\left\langle E_{A}(D) x, x\right\rangle_{\mathrm{H}}$, respectively, $\nu^{x}(D)=\left\langle E_{B}(D) x, x\right\rangle_{\mathrm{H}}$ when $x \in \mathrm{H}$ is arbitrary. With respect to these scalar measures we obtain

$$
\begin{aligned}
a_{n}^{x}=\left\langle A_{n} x, x\right\rangle_{\mathrm{H}} & =\int_{-\infty}^{+\infty} t^{n} \mathrm{~d} \mu^{x}(t) \\
& =\int_{-\infty}^{+\infty} t^{n} \mathrm{~d} \nu^{x}(t), \quad \forall n \in \mathrm{N} .
\end{aligned}
$$

From [5, page 283], the Hamburger scalar moment problem is indeterminate (the sequence $\left\{a_{n}^{x}\right\}_{n}$ does not uniquely determine the scalar representing measure). It follows that the operator-valued representing measure does not uniquely determine the Hamburger operator-valued moment sequence.

However, under some additional conditions about the operator-valued representing measure, the Stieltjes (Hamburger) operator-valued moment sequence is determined [3, pages 509, 510, 511].

Moreover, if the representing measure is that associated with a self-adjoint extension of a symmetric operator with deficiency indices $(0,0)$, the self-adjoint extension is the canonical closure of the given operator and is defined on the whole space. Indeed, if $S: D(S) \rightarrow \mathrm{H}$ is symmetric with $\overline{R(S \pm \mathrm{i})}=\mathrm{H}$ and $A \supset S$, the canonical closure of $S$, it follows that $\mathrm{H} \supseteq R(A \pm \mathrm{i}) \supset R(S \pm \mathrm{i})$ are closed subspaces in $\mathrm{H}$; that is $R(A \pm \mathrm{i})=\mathrm{H}$. In this case the canonical closure of $S$ is the smallest self-adjoint extension of $S$ and is defined on the whole space $\mathrm{H}$ (as in Section 3 of this paper, Proposition 1). The same arguments are in [4, page 1267, Lemma 2.1]. 
Proposition 2. (1) Let $\left\{A_{n}\right\}_{n=0}^{+\infty}, A_{n} \in L(H)$, for all $n \in N, H$ an arbitrary, complex Hilbert space, subject on the conditions $A_{n}=A_{n}^{*}, A_{0}=I d_{H}$, and $E_{1}, E_{2}: \operatorname{Bor}(R) \rightarrow A(H)$ two orthogonal spectral functions on $R$, such that

$$
A_{n}=\int_{-\infty}^{+\infty} t^{n} E_{1}(t)=\int_{-\infty}^{+\infty} t^{n} E_{2}(t), \quad n=0,1,2, \ldots
$$

Then $E_{1}=E_{2}$ on $\operatorname{Bor}(R)$.

Proof. Because $A_{1} \in L(\mathrm{H})$, and $A_{1}=A_{1}^{*}$, the existence of the representation $A_{1}=\int_{-\infty}^{+\infty} t \mathrm{~d} E_{A}(t)$ with $E_{A}: \operatorname{Bor}(\mathrm{R}) \rightarrow$ $A(\mathrm{R}), E_{A}(\mathrm{R})=A_{0}=\mathrm{Id}_{\mathrm{H}}$ is the usual one and is unique. The spectral orthogonal measures coincide; that is, $E_{A}=E_{1}=E_{2}$. The representing measure is the spectral orthogonal measure associated with the self-adjoint operator $A_{1}$. From $E_{A}(\lambda)$ 's multiplicative property, it follows that $A_{n}=\int_{-\infty}^{+\infty} t^{n} E_{A}(t)=$ $\left(\int_{-\infty}^{+\infty} t E_{A}(t)\right)^{n}$ for all $n \in \mathrm{N}$. The uniqueness of the integral representations with respect to spectral functions is assured trivially only in case $A_{n}=A^{n}$, for all $n \in \mathrm{N}$ when the representation is possible.

\section{Stieltjes Operator-Valued Moment Sequences}

A sequence of bounded operators $\Gamma=\left\{\Gamma_{n}\right\}_{n}$, acting on an arbitrary Hilbert space $\mathrm{H}$, is called a Stieltjes operator-valued moment sequence if there exists a positive operator-valued measure $E_{\Gamma}$ on $[0,+\infty)$ such that

$$
\Gamma_{n}=\int_{0}^{+\infty} t^{n} \mathrm{~d} E_{\Gamma}(t), \quad n=0,1,2, \ldots
$$

Proposition 3. Let $\left\{\Gamma_{n}\right\}_{n=0}^{+\infty}$ be an operator sequence with $\Gamma_{n} \in$ $A(H)$ for all $n \in N$ and $\Gamma_{0}=I d_{H}$, with conditions $(A)$ and (B) in Proposition 1 satisfied. The following assertions are equivalent.

(j)

$$
\begin{aligned}
& \text { (1) } \sum_{n, m}\left\langle\Gamma_{n+m} x_{n}, x_{m}\right\rangle_{H} \geq 0 \\
& \text { (2) } \sum_{n, m}\left\langle\Gamma_{n+m+1} x_{n}, x_{m}\right\rangle_{H} \geq 0
\end{aligned}
$$

for all sequences $\left\{x_{n}\right\}_{n=0}^{+\infty} \subset H$ with finite support.

(jj) There exists a positive operator-valued measure $E_{\Gamma}$ : $\operatorname{Bor}(R) \rightarrow A(H)$ such that

$$
\Gamma_{n}=\int_{0}^{+\infty} t^{n} \mathrm{~d} E_{\Gamma}(t), \quad n=0,1,2, \ldots
$$

Proof. We prove that condition (j) [(1) and (2)] is sufficient.

Condition $(j),(1)$ is the same as (i) in Proposition 1. Consequently, there exists a positive operator-valued measure $E_{\Gamma}$ : $\operatorname{Bor}(\mathrm{R}) \rightarrow A(\mathrm{H})$ such that $\Gamma_{n}=\int_{-\infty}^{+\infty} t^{n} \mathrm{~d} E_{\Gamma}(t), n=0,1,2, \ldots$. In the statement $(j),(2)$, if we consider the sequence with finite support $\left\{x_{n}\right\}_{n}$ as $x_{n}=\xi_{n} x, \xi_{n} \in \mathrm{C}, x \in \mathrm{H}$ arbitrary, for all $n \in \mathrm{N}$, we obtain

$$
\begin{aligned}
\sum_{n, m}\left\langle\Gamma_{n+m+1} x_{n}, x_{m}\right\rangle_{\mathrm{H}} & \\
& =\sum_{n, m} \int_{-\infty}^{+\infty} t^{n+m+1} \xi_{n} \overline{\xi_{m}} \mathrm{~d}\left\langle E_{\Gamma}(t) x, x\right\rangle_{\mathrm{H}} \\
& =\int_{-\infty}^{+\infty} t\left|\sum_{n \in I} t^{n} \xi_{n}\right|^{2} \mathrm{~d}\left\langle E_{\Gamma}(t) x, x\right\rangle_{\mathrm{H}} \geq 0,
\end{aligned}
$$

for all polynomials $\sum_{n} t^{n} \xi_{n}$ with complex coefficients and all $x \in \mathrm{H}$. It follows that the representing measure $E_{\Gamma}$ is concentrated on $[0,+\infty)$.

Conversely. The implication $(j j) \Rightarrow(j),(1)$ is the same as (ii) $\Rightarrow$ (i) from Proposition 1 . Moreover, from $(j j)$ it results that, for any sequence $\left\{x_{n}\right\}_{n} \subset \mathrm{H}$ with finite support, we have

$$
\begin{aligned}
\sum_{n, m}\left\langle\Gamma_{n+m+1} x_{n}, x_{m}\right\rangle_{\mathrm{H}} & \\
& =\sum_{n, m} \int_{-\infty}^{+\infty} t^{n+m+1} \mathrm{~d}\left\langle E_{\Gamma}(t) x_{n}, x_{m}\right\rangle_{\mathrm{H}} \\
& =\int_{0}^{+\infty} t \mathrm{~d}\left\langle E_{\Gamma}(t) \sum_{n} t^{n} x_{n}, \sum_{m} t^{m} x_{m}\right\rangle_{\mathrm{H}} \\
& =\int_{0}^{+\infty} t \mathrm{~d}\left\|E_{\Gamma}^{1 / 2}(t) \sum_{n} t^{n} x_{n}\right\| \geq 0,
\end{aligned}
$$

that is, $(j),(2)$.

We give another second characterization on an operator sequence $\left\{\Gamma_{n}\right\}_{n=0}^{+\infty}$ to be an operator-valued Stieltjes moment sequence.

Remark 4. In the sequel, we argue like in [1, page 329]. Between the Hamburger operator-valued moment sequences and Stieltjes moment sequences we can establish the following bijection.

(A) If $\Gamma=\left\{\Gamma_{n}\right\}_{n=0}^{+\infty}$ is a Stieltjes moment sequence with respect to the spectral measure $E_{\Gamma}(t)$ on $[0,+\infty)$ for the homeomorphism $\pi_{1}:[0,+\infty) \rightarrow(-\infty, 0], \pi_{1}(t)=$ $-\sqrt{t}$, there corresponds a spectral measure $F_{\Gamma}^{1}$ on $(-\infty, 0]$ defined by $F_{\Gamma}^{1}(t)=(1 / 2)\left[E_{\Gamma}(\infty)-E_{\Gamma} \circ \pi_{1}^{-1}(t)\right]$ such that $\int_{-\infty}^{0} \lambda^{2 k} \mathrm{~d} F_{\Gamma}^{1}(\lambda)=(1 / 2) \int_{0}^{+\infty} \lambda^{k} \mathrm{~d} E_{\Gamma}$.

For the homeomorphism $\pi_{2}:[0,+\infty) \rightarrow[0,+\infty)$, $\pi_{2}(t)=\sqrt{t}$, there corresponds a spectral measure $F_{\Gamma}^{2}$ on $[0,+\infty)$ defined by $F_{\Gamma}^{2}(t)=(1 / 2) E_{\Gamma}(\infty)+(1 / 2) E_{\Gamma} \circ \pi_{2}^{-1}(t)$ such that $\int_{0}^{\infty} \lambda^{2 k} \mathrm{~d} F_{\Gamma}^{2}(\lambda)=(1 / 2) \int_{0}^{+\infty} \lambda^{k} \mathrm{~d} E_{\Gamma}$. 
We define

$$
F_{\Gamma}= \begin{cases}F_{\Gamma}^{1}(t), & t<0, \\ F_{\Gamma}^{2}(t), & t \geq 0 .\end{cases}
$$

For $F_{\Gamma}(t)$ we have the representations: $\int_{-\infty}^{+\infty} \lambda^{2 k} \mathrm{~d} F_{\Gamma}(\lambda)=$ $\int_{0}^{+\infty} \lambda^{k} \mathrm{~d} E_{\Gamma}(\lambda), k=0,1, \ldots, n, \ldots$ and $\int_{-\infty}^{+\infty} \lambda^{2 k+1} \mathrm{~d} F_{\Gamma}(\lambda)=0$, $k=0,1, \ldots, n, \ldots$.

(B) Conversely. If we have the operator-valued Hamburger moment sequence $\left\{\Gamma_{0}, 0, \Gamma_{1}, 0, \Gamma_{2}, 0, \ldots\right\}$ with respect to the spectral representing measure $F_{\lambda}$, respectively, the sequence $\left\{B_{n}\right\}_{n=0}^{+\infty}$, defined by $B_{n}=\left(\Gamma_{[n / 2]}+(-1)^{n} \Gamma_{[n / 2]}\right) / 2$ for all $n \in \mathrm{N}$, admits the integral representation $B_{2 n}=\Gamma_{n}=$ $\int_{-\infty}^{+\infty} t^{2 n} \mathrm{~d} F(\lambda)$ and $B_{2 n+1}=0=\int_{-\infty}^{+\infty} t^{2 n+1} \mathrm{~d} F(t)$. We can construct a spectral measure $E(t)$ on $[0,+\infty)$; that is, for $\pi$ : $(-\infty,+\infty) \rightarrow[0,+\infty), \pi(t)=t^{2}$ we have $E(t)=F \circ \pi^{-1}(t)$ with $\int_{-\infty}^{+\infty} t^{n} \mathrm{~d} F(t)=\int_{0}^{+\infty}\left(t^{2}\right)^{n} \mathrm{~d} E(t)=B_{2 n}=\Gamma_{n}, n=0,1,2, \ldots$.

For obtaining positive operator-valued measures from orthogonal, spectral functions, in both cases, we compose the orthogonal spectral measure associated with a self-adjoin operator with the same projections, respectively, $h_{0}, h_{0}^{*}$ in our case. Summing the above conditions (A) and (B) in Remark 4, for an operator-valued sequence $\left\{\Gamma_{n}\right\}_{n=0}^{\infty} \in L(\mathrm{H})$, we construct the operator-valued sequence $B_{n}=\left(\Gamma_{[n / 2]}+\right.$ $\left.(-1)^{n} \Gamma_{[n / 2]}\right) / 2$ for all $n \in N$ and, conversely, for a sequence $\left\{B_{n}\right\}_{n=0}^{+\infty} \in L(\mathrm{H})$, we construct the operator-valued sequence $\left\{\Gamma_{n}\right\}_{n=0}^{+\infty}$, with $\Gamma_{n}=B_{2 n}, n \in \mathrm{N}$.

With the above construction, we have the following.

Proposition 5. The sequence $\left\{\Gamma_{n}\right\}_{n=0}^{+\infty}$ that satisfies conditions (A) and (B) in Proposition 1 is a Stieltjes operator-valued moment sequence if and only if

$$
\sum_{n, m=0}^{q}\left\langle\left(\frac{\Gamma_{[(n+m) / 2]}+(-1)^{n+m} \Gamma_{[(n+m) / 2]}}{2}\right) x_{n}, x_{m}\right\rangle_{H} \geq 0
$$

for all sequences $\left\{x_{n}\right\}_{n=0}^{+\infty} \subset H$ with finite support and all $q \in N$.

Proposition $5^{\prime}$ (reformulated). The sequence $\left\{\Gamma_{n}\right\}_{n=0}^{+\infty}$ is a Stieltjes operator-valued moment sequence if and only if

$$
\sum_{n, m=0}^{q}\left\langle B_{n+m} x_{n}, x_{m}\right\rangle_{\mathrm{H}} \geq 0
$$

for all sequences $\left\{x_{n}\right\}_{n=0}^{+\infty} \subset \mathrm{H}$ with finite support and all $q \in \mathrm{N}$, $B_{n}$, for all $n \in \mathrm{N}$ defined above.

Proof. Let $\left\{\Gamma_{n}\right\}_{n=0}^{+\infty}$ be an operators' sequence, $\Gamma_{n} \in L(\mathrm{H})$, $\mathrm{H}$ an arbitrary complex Hilbert space; we define $B_{n}=$ $\left(\Gamma_{[n / 2]}+(-1)^{n} \Gamma_{[n / 2]}\right) / 2, n \in \mathrm{N}$; that is, $B_{2 n}=\Gamma_{n}$ and $B_{2 n+1}=0$, for all $n \in \mathrm{N}$. In the condition of the hypothesis (Propositions 5 and $5^{\prime}$ ), we have $\sum_{n, m}\left\langle B_{n+m} x_{n}, x_{m}\right\rangle_{\mathrm{H}} \geq 0$ for all sequences $\left\{x_{n}\right\}_{n} \subset \mathrm{H}$ with finite support. From
Proposition 1, there exists a positive operator-valued measure on $\mathrm{R}$ such that

$$
B_{n}=\int_{-\infty}^{+\infty} t^{n} \mathrm{~d} F(t), \quad n=0,1,2, \ldots
$$

From Remark 4, there exists a positive operator-valued measure $E(t)$ such that

$$
\begin{array}{r}
B_{2 n}=\int_{-\infty}^{+\infty} t^{2 n} \mathrm{~d} F(t)=\Gamma_{[2 n / 2]}=\Gamma_{n}=\int_{0}^{+\infty} t^{n} \mathrm{~d} E(t), \\
n=0,1,2, \ldots .
\end{array}
$$

That is $\left\{\Gamma_{n}\right\}_{n \in \mathrm{N}}$ is a Stieltjes operator-valued moment sequence.

Conversely. If $\Gamma_{n}=\int_{0}^{+\infty} t^{n} \mathrm{~d} E(t), n=0,1,2, \ldots$, we construct a measure $F(t)$ positively defined on $(-\infty,+\infty)$, as in Remark 4, with the property that $\Gamma_{n}=\int_{-\infty}^{+\infty} t^{2 n} \mathrm{~d} F(t)=$ $\int_{0}^{+\infty} t^{n} \mathrm{~d} E(t)=B_{2 n}$; and $B_{2 n+1}=\int_{-\infty}^{+\infty} t^{2 n+1} \mathrm{~d} F(t)=0$. In this case,

$$
\begin{gathered}
\sum_{n, m=0}^{q}\left\langle\left(\frac{\Gamma_{[(n+m) / 2]}+(-1)^{n+m} \Gamma_{[(n+m) / 2]}}{2}\right) x_{n}, x_{m}\right\rangle_{\mathrm{H}} \\
\quad=\sum_{n, m=0}^{q}\left\langle\int_{-\infty}^{+\infty} t^{n+m} \mathrm{~d} F(t) x_{n}, x_{m}\right\rangle_{\mathrm{H}} \\
=\int_{-\infty}^{+\infty} \mathrm{d}\left\|\sum_{n} t^{n} F^{1 / 2}(t) x_{n}\right\|^{2} \geq 0 .
\end{gathered}
$$

Proposition 6. Let $\left\{\Gamma_{n}\right\}_{n=0}^{+\infty}$ be an operator sequence with $\Gamma_{n} \in$ $A(H)$ for all $n \in N$ and $\Gamma_{0}=I d_{H}$. The following assertions are equivalent:

(j)

$$
\begin{aligned}
& \text { (1) } \sum_{n, m}\left\langle\Gamma_{n+m} x_{n}, x_{m}\right\rangle_{H} \geq 0 \\
& \text { (2) } \sum_{n, m}\left\langle\Gamma_{n+m+1} x_{n}, x_{m}\right\rangle_{H} \geq 0
\end{aligned}
$$

for all sequences $\left\{x_{n}\right\}_{n=0}^{+\infty} \subset H$ with finite support.

(jj) There exists a positive operator-valued measure $E_{\Gamma}$ : $\operatorname{Bor}(R) \rightarrow A(H)$ such that

$$
\Gamma_{n}=\int_{0}^{+\infty} t^{n} \mathrm{~d} E_{\Gamma}(t), \quad n=0,1,2, \ldots
$$

Proof. We prove that condition ( $j)[(1)$ and (2)] is sufficient.

Condition ( $j),(1)$ is the same with (i) in Proposition 1. Consequently, there exists a positive operator-valued measure $E_{\Gamma}: \operatorname{Bor}(\mathrm{R}) \rightarrow A(\mathrm{H})$ such that $\Gamma_{n}=\int_{-\infty}^{+\infty} t^{n} \mathrm{~d} E_{\Gamma}(t)$, $n=0,1,2, \ldots$ In the statement $(j),(2)$, if we consider the 
sequence with finite support $\left\{x_{n}\right\}_{n}$ as $x_{n}=\xi_{n} x, \xi_{n} \in \mathrm{C}, x \in \mathrm{H}$ arbitrary, for all $n \in \mathrm{N}$, we obtain

$$
\begin{aligned}
\sum_{n, m}\left\langle\Gamma_{n+m+1} x_{n}, x_{m}\right\rangle_{\mathrm{H}} & \\
= & \sum_{n, m} \int_{-\infty}^{+\infty} t^{n+m+1} \xi_{n} \overline{\xi_{m}} \mathrm{~d}\left\langle E_{\Gamma}(t) x, x\right\rangle_{\mathrm{H}} \\
& =\int_{-\infty}^{+\infty} t\left|\sum_{n \in I} t^{n} \xi_{n}\right|^{2} \mathrm{~d}\left\langle E_{\Gamma}(t) x, x\right\rangle_{\mathrm{H}} \geq 0,
\end{aligned}
$$

for all polynomials $\sum_{n} t^{n} \xi_{n}$ with complex coefficients and all $x \in \mathrm{H}$. It follows that the representing measure $E_{\Gamma}$ is concentrated on $[0,+\infty)$.

Conversely. The implication $(j j) \Rightarrow(j),(1)$ is the same with (ii) $\Rightarrow$ (i) from Proposition 1 . Moreover, from $(j j)$ it results that, for any sequence $\left\{x_{n}\right\}_{n} \subset \mathrm{H}$ with finite support, we have

$$
\begin{aligned}
\sum_{n, m}\left\langle\Gamma_{n+m+1} x_{n}, x_{m}\right\rangle_{\mathrm{H}} & \\
= & \sum_{n, m} \int_{-\infty}^{+\infty} t^{n+m+1} d\left\langle E_{\Gamma}(t) x_{n}, x_{m}\right\rangle_{\mathrm{H}} \\
& =\int_{0}^{+\infty} t \mathrm{~d}\left\langle E_{\Gamma}(t) \sum_{n} t^{n} x_{n}, \sum_{m} t^{m} x_{m}\right\rangle_{\mathrm{H}} \\
& =\int_{0}^{+\infty} t \mathrm{~d}\left\|E_{\Gamma}^{1 / 2}(t) \sum_{n} t^{n} x_{n}\right\| \geq 0,
\end{aligned}
$$

that is $(j),(2)$.

\section{Conflict of Interests}

The author declares that there is no conflict of interests regarding the publication of this paper.

\section{References}

[1] T. Andô, "Truncated moment problems for operators," Acta Scientiarum Mathematicarum, vol. 31, pp. 319-334, 1970.

[2] J. W. Helton and M. Putinar, "Positive polynomials in scalar and matrix variables, the spectral theorem, and optimization," in Operator Theory, Structured Matrices, and Dilations, vol. 7, pp. 229-307, Theta, Bucharest, Romania, 2007.

[3] F. J. Narcowich, " $R$-operators. II. On the approximation of certain operator-valued analytic functions and the Hermitian moment problem," Indiana University Mathematics Journal, vol. 26, no. 3, pp. 483-513, 1977.

[4] F.-H. Vasilescu, "Hamburger and Stieltjes moment problems in several variables," Transactions of the American Mathematical Society, vol. 354, no. 3, pp. 1265-1278, 2002.

[5] G. Choquet, Lectures on Analysis, vol. 2, Benjamin, New York, NY, USA, 1969. 


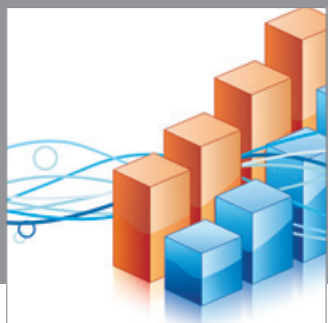

Advances in

Operations Research

mansans

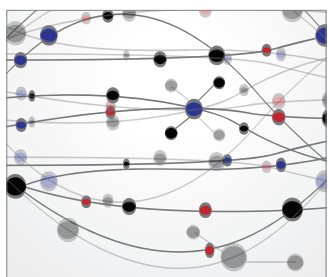

The Scientific World Journal
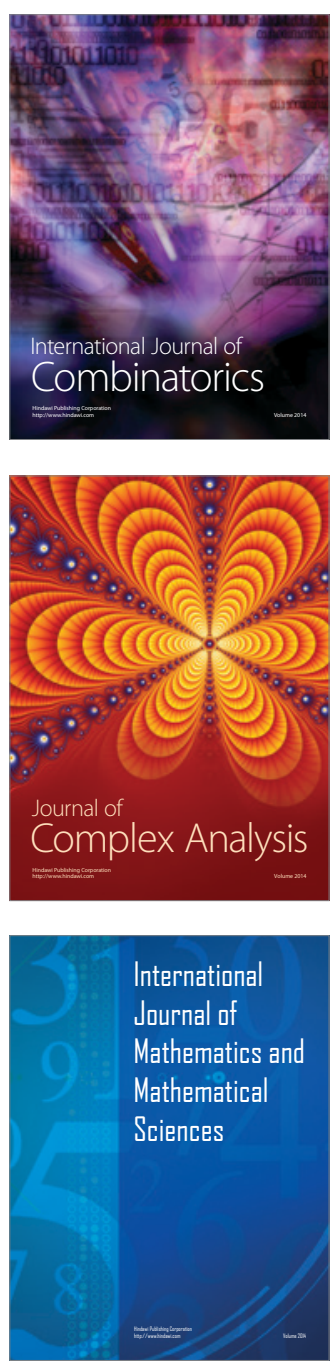
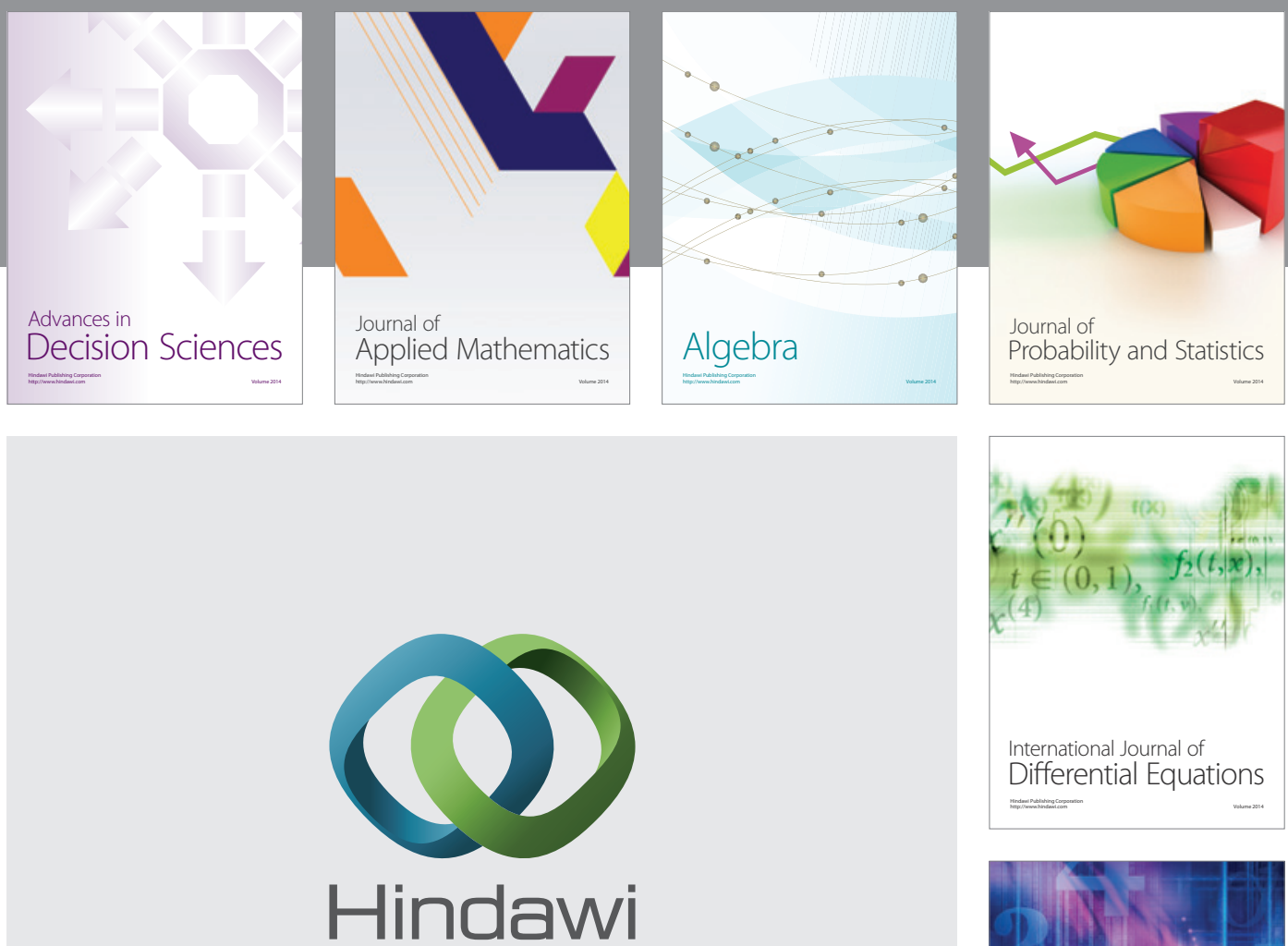

Submit your manuscripts at http://www.hindawi.com
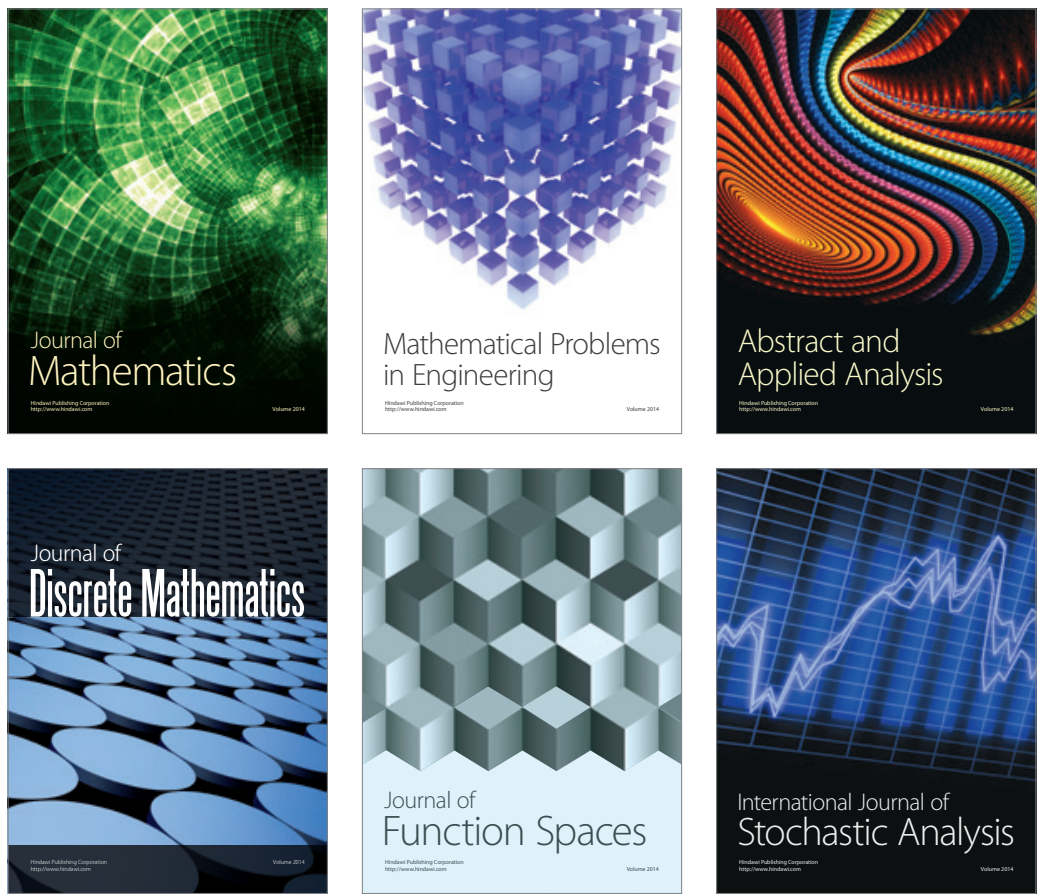

Journal of

Function Spaces

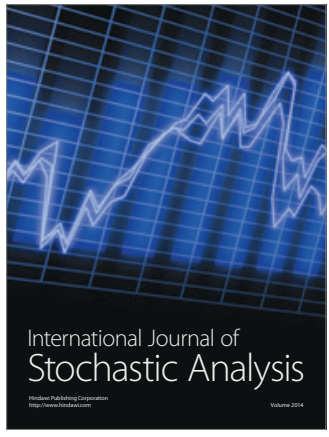

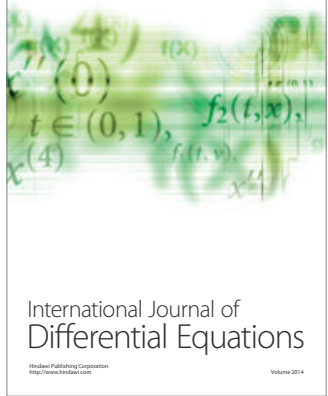
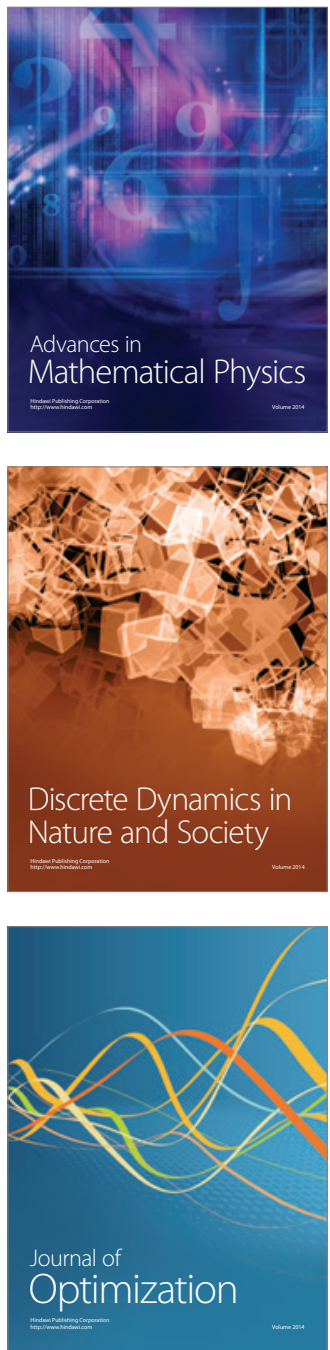